\title{
Varón de 48 años con diarrea y colestasis
}

\author{
48 year old male with diarrhea and cholestasis
}

\author{
A. Jiménez Jurado, G. Alonso Claudio \\ Servicio de Medicina Interna. Hospital Clínico Universitario de Salamanca. SACYL. Salamanca
}

\begin{abstract}
Resumen
Presentamos el caso de un varón de 48 años derivado a la consulta por un cuadro clínico de diarrea crónica (> 6 semanas) junto con alteración de las pruebas de función hepática consistente en un patrón de colestasis. La presencia de diarrea crónica y colestasis nos obligaron al diagnóstico diferencial entre las siguientes entidades: enfermedad celíaca, enfermedad inflamatoria intestinal, enfermedades infecciosas crónicas (tuberculosis intestinal, micobacterium avium en inmunodeprimidos), causas metabólicas como la amiloidosis, obstrucción de la vía biliar intrahepatica (ductopenia del adulto, colangitis esclerosante primaria o secundaria), toma de fármacos y finalmente causas tumorales (colangiocarcinoma, linfoma de Hodgkin, adenocarcinoma colon). En el caso que presentamos, la colangio resonancia magnética nuclear fue la herramienta diagnóstica fundamental para el diagnóstico de la entidad.
\end{abstract}

Palabras clave. Colangitis esclerosante primaria. Diarrea crónica. Colangio resonancia magnética. Colitis ulcerosa.

\section{Introducción}

La colangitis esclerosante primaria es una enfermedad colestásica crónica caracterizada por la inflamación y fibrosis de las vías biliares intrahepáticas y extrahepáticas, que puede evolucionar hasta originar una cirrosis biliar. La etiología de esta patología es desconocida, aunque se postula la hipótesis de predisposición a fenómenos autoinmunes junto con una participación de factores ambientales.

Se asocia frecuentemente a enfermedad inflamatoria intestinal, especialmente a colitis ulcerosa, por ello debemos sospecharla ante la presencia analítica de un patrón de colestasis en un paciente con dicha enfermedad.

Presentamos a continuación el caso de un paciente de 48 años con un cuadro clínico consistente en diarrea crónica, pérdida de peso y alteración persistente del perfil hepático.

\section{Caso clínico}

Varón de 48 años de edad, sin alergias farmacológicas conocidas, con el antecedente de un angiosarcoma cutáneo de hombro izquierdo intervenido en 1997. Acudió a la consulta de Medicina Interna derivado por su médico de Atención Primaria por un cuadro de diarrea y alteración del perfil hepático. El paciente refería desde hacía años entre 3-5 deposiciones al día de consistencia líquida sin productos patológicos aunque en los últimos 6 meses presentó aumento del número y cantidad de las deposiciones, ocasionalmente con sangre roja y pérdida de peso, sin pérdida de apetito. El cuadro clínico no se acompañaba de náuseas, vómitos o dolor abdominal. Negaba fiebre. En la exploración física destacaba un IMC de $17 \mathrm{~kg} / \mathrm{m} 2$, el resto era normal.

En la analítica presentaba una $\mathrm{Hb}: 12.4 \mathrm{gr} / \mathrm{dl}$ con leucocitos y plaquetas normales. La función renal e iones eran normales. La ferritina fue de 26ng/ml, con resto del estudio del hierro normal. En el perfil hepático destacaba una bilirrubina normal con AST: 101 UI/L, ALT: 140 UI/L,

\begin{abstract}
We report the case of a 48 year old male referred for consultation regarding chronic diarrhea (> 6 weeks) with alteration of liver function tests consisting of a pattern of cholestatic pattern. The presence of diarrea and chronic cholestasis, forced us to make a differential diagnosis between the following entities: celiac disease, inflammatory bowel disease, chronic infectious diseases (intestinal tuberculosis, Mycobacterium avium in immunocompromised patient), metabolic causes such as amyloidosis, obstruction of intrahepatic bile duct (adult ductopenia, secondary or primary sclerosing cholangitis), drugs and eventually neoplastic causes (cholangiocarcinoma, Hodgkin's lymphoma, colon adenocarcinoma). In the case presented, nuclear magnetic resonance cholangiopancreatography was the key diagnostic tool for the diagnosis of the entity.
\end{abstract}

Key words. Primary sclerosing cholangitis. Chronic diarrhea. Magnetic resonance cholangiopancreatography. Ulcerative colitis

FA: 1137 UI/L, GGT: 516 UI/L. En el proteinograma presentaba unas gammaglobulinas de 2.19gr/dl. La grasa en heces fue de $20.10 \mathrm{~g} / 24$ horas (normal 0-6).Los anticuerpos anti-músculo liso, los anticuerpos anti-mitocondriales, el estudio de autoinmunidad, así como los marcadores tumorales, fueron normales.

En cuanto a la microbiología, las serologías para VIH, VHB, VHC y CMV resultaron negativas, así como los cultivos de heces para bacterias, parásitos y micobacterias.

Se realizó al paciente una colonoscopia desde recto hasta ciego en la que se observó un intenso edema, hiperemia, úlceras superficiales coalescentes en algunos tramos con sangrado al roce. Se tomaron múltiples biopsias y el resultado anatomopatológico definitivo fue de pancolitis ulcerosa con actividad moderada-grave.

El estudio se completó con una colangio resonancia magnética nuclear en la cual se visualizó estenosis del colédoco intrapancreático $(3 \mathrm{~cm})$ sin apreciarse lesión parenquimatosa dependiente de la cabeza del páncreas. Además se vio dilatación del colédoco proximal, de ambos hepáticos y de los radicales biliares intrahepáticos izquierdos, que se

Figura 1. Colangioresonancia magnética nuclear. Patrón radiologógico típico consistente en múltiples estenosis irregulares y dilataciones segmentarias de los conductos biliares intrahepáticos.

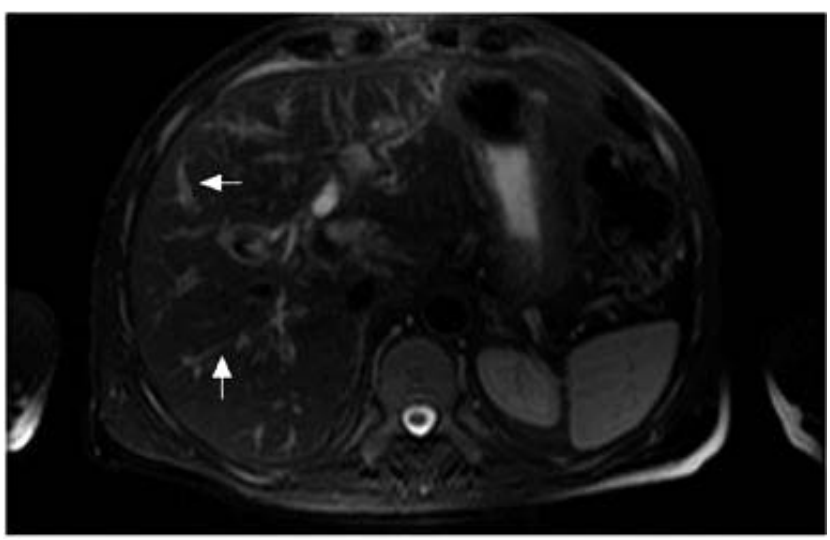


mostraban discretamente arrosariados (figura 1). Estos hallazgos descritos fueron compatibles con el diagnóstico de colangitis esclerosante.

\section{Discusión}

La colangitis esclerosante primaria (CEP) es una enfermedad colestásica crónica caracterizada por la inflamación, fibrosis y estenosis de los conductos biliares intra y/o extrahepáticos, que conducen a colestasis, cirrosis hepática, hipertensión portal, y en última instancia, muerte prematura por fallo hepático ${ }^{1}$.

La prevalencia es muy variable según diversos estudios epidemiológicos, siendo altamente prevalente en los países escandinavos, donde representa la primera causa de trasplante hepático. Es mucho menos frecuente en países como España, Italia, India o Japón. Las tasas de incidencia y prevalencia son superiores en varones ${ }^{2}$ y suele aparecer en la tercera década de la vida. La prevalencia de la enfermedad inflamatoria intestinal (EII) encontrada en la CEP es alta, estando presente en aproximadamente el 70\% de los casos, existiendo una gran variación entre estudios. El tipo más común es la colitis ulcerosa (CU), que representa más del $75 \%$ de la Ell encontrada en la CEP, seguida por la enfermedad de Crohn (EC) y por la infrecuente colitis indeterminada 3 .

La etiología es desconocida sin embargo se han propuesto diferentes factores genéticos y adquiridos, tóxicos o infecciosos, que podrían desempeñar un papel clave en la fisiopatogenia. Se ha descrito un infiltrado inflamatorio por linfocitos T con aumento de la expresión de factor de necrosis tumoral alfa (TNF- ) y cambios en los colangiocitos que expresan citocinas proinflamatorias y profibrogénicas y moléculas de adherencia 4 . Se produciría una fibrosis concéntrica con atrofia del endotelio biliar secundario a la isquemia. La pérdida paulatina de los conductos biliares produciría colestasis progresiva, fibrosis y cirrosis biliar.

Se debe sospechar una CEP en todo paciente con colestasis e historia previa de Ell. Las manifestaciones clínicas son muy variables. Los pacientes con CEP pueden estar asintomáticos y ser diagnosticados como parte de la evaluación de pruebas de laboratorio anormales, o pueden tener síntomas como fatiga 0 prurito. El examen físico puede revelar ictericia, hepatomegalia, esplenomegalia y excoriaciones, aunque a menudo es normal. En ocasiones, la enfermedad se diagnostica en estadios avanzados cuando hay hipertensión portal con ascitis o hemorragia digestiva por várices esofágicas.

Las determinaciones de laboratorio revelan un patrón de colestasis con un aumento de la fosfatasa alcalina, la gammaglutamiltransferasa y un aumento moderado de las transaminasas. La bilirrubinemia, la albuminemia y la tasa de protrombina son normales en el momento del diagnóstico hasta en el 60\% de los casos. Puede observarse un aumento de las inmunoglobulinas, predominantemente de lgG, en el $61 \%$ de los casos. Los anticuerpos antimitocondriales son negativos. Desde el punto de vista inmunológico, el dato más característico es la detección de anticuerpos contra los neutrófilos con un patrón perinuclear (p-ANCA) que se observa en el 26-85\% de los casos, aunque carecen de especificidad para el diagnóstico de CEP.

El método más preciso para el diagnóstico es la colangiografía retrógrada endoscópica (CPRE) pero esta exploración invasiva se asocia a una tasa no despreciable de complicaciones infecciosas y pancreatitis. Debido a que la colangio resonancia magnética nuclear es no invasiva y presenta una precisión diagnóstica com- parable a la CPRE, es típicamente la primera prueba elegida ${ }^{5}$.Se realizará la CPRE cuando se contemple una actuación terapéutica. El patrón radiológico típico consiste en múltiples estenosis irregulares y dilataciones segmentarias de los conductos biliares intra y/o extrahepáticos.

La biopsia hepática rara vez proporciona información complementaria que condicione el tratamiento de los pacientes con CEP6, por lo que no se aconseja realizarla de forma sistemática. Está especialmente indicada ante la sospecha de un síndrome de superposición con una hepatitis autoinmune, cuando los pacientes presentan manifestaciones de las dos enfermedades o ante la sospecha de CEP de pequeños conductos con colangiografía normal. El tratamiento específico consiste en administrar ácido ursodesoxicólico (AUDC). A una dosis de 20 mg/kg de peso, el tratamiento tiene efectos favorables y mejora las alteraciones analíticas, retrasa la progresión de la fibrosis y mejora las anomalías colangiográficas; sin embargo no existe evidencia definitiva de que mejore la supervivencia y sea eficaz en la prevención de neoplasia colorrectal-biliar. Avances recientes en la comprensión de los mecanismos patológicos que contribuyen al daño hepatobiliar en la CEP han permitido el desarrollo de ensayos clínicos para evaluar posibles candidatos como herramientas terapéuticas en CBP. De esta manera, se están estudiando posibles tratamientos como los miméticos de ácidos biliares, agonistas del receptor $X$ de farnesonida, inhibidores de los transportadores de ácidos biliares apicales sodio-dependientes, determinados antibióticos y anticuerpos monoclonales ${ }^{7}$.

En relación al trasplante hepático, la mayoría de los grupos están de acuerdo en que se debe indicar cuando la bilirrubinemia se mantiene $>6 \mathrm{mg} / \mathrm{dl}$ durante más de 6 meses, aparecen complicaciones de la hipertensión portal como hemorragia por varices, ascitis o encefalopatía hepática), aparecen episodios recurrentes de colangitis bacteriana y/o ante el prurito refractario al tratamiento médico.

El pronóstico es inferior a la de la población general. Se ha observado una supervivencia media de 18 años en los pacientes asintomáticos y de 8,5 años en los sintomáticos desde el diagnóstico de la enfermedad. En algunos casos puede causar la muerte por insuficiencia hepática o por el desarrollo de un colangiocarcinoma (entre un 8-18\% de los pacientes con CEP desarrollan esta neoplasia) comportando un muy mal pronóstico con una supervivencia media inferior al año.

\section{Bibliografía}

1. Tsaitas $C$, Semertzidou A, Sinakos E. Update on inflammatory bowel disease in patients with primary sclerosing cholangitis. World J Hepatol. 2014;6:178-87.

2. Bambha K, Kim WR, Talwalkar J, Torgerson H, Benson JT, Therneau TM et al. Incidence, clinical spectrum, and outcomes of primary sclerosing cholangitis in a United States community.Gastroenterol. 2003;125:1364-9.

3. De Vries AB, Janse M, Blokzijl H, Weersma RK. Distinctive inflammatory bowel disease phenotype in primary sclerosing cholangitis. World $\mathrm{J}$ Gastroenterol. 2015;21:1956-71.

4. Parés A. Colangitis esclerosante primaria: diagnóstico, pronóstico y tratamiento. Gastroenterol. Hepatol. 2011;34:41-52.

5. Dave M, Elmunzer BJ, Dwamena BA, Higgins PD. Primary Sclerosing Cholangitis: Meta-Analysis of Diagnostic Performance of MR Cholangiopancreatography. Radiology. 2010;256:387-96.

6. Burak KW, Angulo P, Lindor KD. Is there a role for liver biopsy in primary sclerosing cholangitis? Am J Gastroenterol. 2003;98:1155-8.

7. Ahmad H Ali, Elizabeth J Carey, Keith D Lindor. Current research on the treatment of primary sclerosing colangitis. Intractable Rare Dis Res. 2015;4:1-6. 